\title{
HERMITE-HADAMARD INEQUALITY FOR POINT-WISE CONVEX MAPS AND LEGENDRE-FENCHEL CONJUGATION
}

\author{
SEVER S. DRAGOMIR AND Mustapha RAÏSSOUli
}

Abstract. In the present paper, Hermite-Hadamard inequality for point-wise convex maps is given. Jensen type inequality for the Legendre-Fenchel conjugation is also established. Applications are given to the case of quadratic functionals and positive operators.

Mathematics subject classification (2010): 26D10, 26D15, 47A63, 47A64, 46S20.

Keywords and phrases: Hermite-Hadamard inequality, point-wise convex maps, convex analysis, LegendreFenchel conjugation, operator and functional means.

\section{REFERENCES}

[1] S. S. Dragomir, Two mappings in connection to Hadamard's inequalities, J. Math. Anal. Appl., 167 (1992), 42-56.

[2] S. S. DRAGOMIR AND A. MCANDREW, Refinements of the Hermite-Hadamard inequality for convex functions, J. Inequal. Pure and Appl. Math., 6 (5)(2005), Art. 140

[3] S. S. Dragomir AND M. Raïssouli, Iterative Refinements of the Hermite-Hadamard Inequality, Applications to Standard Means, J. Ineq. App. Vol. 2010 (2010), Article ID 107950, 13 pages.

[4] P. J. Laurent, Approximation et Optimisation, Herman, 1972.

[5] R. D. Nussbaum And J. E. Cohen, The Arithmetic-Geometric Mean and Its Generalizations for Noncommuting Linear Operators, An. Sc. Norm. Sup. Sci (4) 15, No. 2 (1989), 239-308.

[6] M. RaÏssouli And H. Bouziane, Functional Logarithm in the sense of Convex Analysis, J. Conv. An., Vol. 10 (2003), No. 1, 229-244.

[7] M. Raïssouli and H. Bouziane, Arithmetico-Geometrico-Harmonic Functional Mean in the sense of Convex Analysis, Ann. Sc. Math. Québec, Vol. 3 (2006), No. 1, 79-107.

[8] M. RaÏssouli, Logarithmic Functional Mean in Convex Analysis, J. Ineq. Pure App. Math., Vol. 10 (2009), Issue 4, Article 102.

[9] M. RaÏssouli, Discrete Operator and Functional Means ca be reduced to the Continuous Arithmetic Mean, Int. J. Open Prob. Comp. Sc. Math., Vol. 3, No. 2 (2010), 186-199.

[10] M. RAÏssouli, United Explicit Form for a Game of Monotone and Chaotic Matrix Means, Int. Elec. J. Pure App. Math., Vol. 1, No. 4 (2010), 475-493.

[11] E. ZEIDLER, Nonlinear Functional Analysis and its Applications III, Springer-Verlag, 1984. 